\title{
Scanning, holistic encoding, and the word-superiority effect
}

\author{
GARVIN CHASTAIN \\ Boise State University, Boise, Idaho 83725
}

\begin{abstract}
The role of holistic encoding induced by scanning in the superior identifiability target letters in orthographic strings (words) enjoy over those in strings of unrelated letters was examined in two experiments. Both the set of potential targets and the critical position in the letter arrays were predesignated. One group in each experiment was induced to scan each array from left to right in a dot-counting task. A dot could precede the array to its left and/or appear with the array to its right. Only this group displayed the word superiority. For the second group in the first experiment, any dot(s) present always appeared with the array. The second group in the second experiment was shown no dots but, rather, performed the letter-identification task only. The absence of a word-superiority effect in other studies is related to holistic encoding stemming from scanning requirements, and the implications for positional uncertainty explanations of the phenomenon are discussed.
\end{abstract}

A letter in a word can be identified more accurately than one in a string of unrelated letters if the exposure is brief and followed by a noise or pattern mask. The superior accuracy on letters in words extends to all orthographic strings, whether or not they have lexical status (Baron \& Thurston, 1973; Manelis, 1974); thus, for present purposes, all orthographic strings will be considered words. The word-superiority effect (WSE) occurs when the set of target letters is predesignated (Carr, Lehmkuhle, Kottas, Astor-Stetson, \& Arnold, 1976; Chastain, 1981; Spector \& Purcell, 1977). However, if the position of the target in the string is held constant across trials, the WSE has usually been found to disappear (Johnston \& McClelland, 1974; Massaro, 1973; Paap \& Newsome, 1980a). Paap and Newsome (1980b) presented the target letter in different positions in the string, but they found that a cue designating the position of the target before each exposure eliminated the WSE. Accordingly, Chastain (1977) used a repeatedexposures procedure (Haber \& Hershenson, 1965) and found that eight CVCs were identified no more rapidly than eight trigrams consisting of all consonants. Analysis of the order in which the letters were identified indicated that subjects concentrated on one position at a time, identifying the letter in a given position before proceeding to the next one to the right. Smith and Spoehr (1974) suggested that prior information about the position of the target letter causes subjects to ignore the other letters in the string and thereby eliminates the benefits of the word context.

Nevertheless, Chastain (1981) observed a WSE when trigrams (with the middle letter always designated as the

Requests for reprints should be addressed to Garvin Chastain, Department of Psychology, Boise State University, Boise, Idaho 83725 . target) became quadrigrams with the addition of a terminal letter from the postexposure mask. Different terminal letters were combined with the original trigrams to produce orthographic and nonorthographic quadrigrams. Greenburg and Krueger (1980) also reported a WSE with a predesignated pair of confusable target letters appearing in a predesignated position in trigrams, but only when subjects were explicitly encouraged to attend to the entire trigram.

A possible answer to the question of exactly what subjects are doing to produce the WSE when encouraged to attend to the entire trigram is suggested by the procedure used by Chastain (1981). Although subjects in that study were not told to consider the entire letter array, the appearance of different terminal letters with the postexposure mask may have induced the subjects to scan across the array from left to right to discover which terminal letter would complete each exposure. Since subjects self-initiated each presentation, the scan might have been accomplished with an eye movement, or it could have been an internal scan of a posticonic buffer (Mewhort \& Beal, 1977; Mewhort, Merikle, \& Bryden, 1969). Due to the subjects' experiences in reading, scanning across the array in this manner may have induced them to attempt to encode the entire letter string holistically. A substantial amount of evidence has accumulated to suggest that letter strings that constitute words can be encoded holistically without the preliminary identification of each letter in the string (Jacewicz, 1979; Johnson \& Marmurek, 1978; Lawry \& Laberge, 1981; McClelland, 1979); nonetheless, a given letter in such a string enjoys an advantage in identifiability over one in a string that cannot be so encoded (McClelland \& Rumelhart, Note 1; Rumelhart \& McClelland, Note 2). If a scan producing holistic encoding of strings constituting words is required for 
the WSE to occur with targets in a constant position in the array, presenting stimuli in a manner that requires a left-to-right scan should produce the WSE, but presenting the same stimuli in a way that requires no such scan should not. This prediction was tested in two experiments.

\section{EXPERIMENT 1}

Purcell, Stanovich, and Spector (1978) found a WSE using $\mathrm{A} \_\mathrm{E}$ as word strings and $\mathrm{V} \_\mathrm{H}$ as unrelated letter strings, with $C, G, P$, and $R$ as possible targets in the middle position. However, Paap and Newsome (1980a) placed selected digits $(3,6,8$, or 9$)$ between the same sets of flanking letters and found significantly lower performance with the $\mathrm{V}$ and $\mathrm{H}$ flankers, leading them to conclude that the original effect was due to differential lateral masking. This potential confounding can be overcome with arrays composed of targets and flankers that are each bilaterally symmetrical across the vertical midline, with the two relative positions of the flankers reversing to form words and nonwords. Accordingly, letter arrays in the current experiment were TAX, TUX, XAT, and XUT. Identification of the target letter in the middle was the secondary task. The primary task was counting the number of dots that flanked the letter array on each exposure. A dot could precede, follow, both precede and follow, or neither precede nor follow the letters, for a total of zero, one, or two dots. For one group of subjects, the dot to the left, if present, always appeared first, followed by a brief interval during which the preexposure mask returned, followed by the letters with possibly a dot to the right. For the other group of subjects, any $\operatorname{dot}(\mathrm{s})$ present always appeared simultaneously with the letters. Due to the nature of the presentation, the former group would be induced to scan the array from left to right, whereas the latter group would not. If an attempt to encode a string in a holistic manner, which can be induced by the left-toright scan used in reading, is important to the WSE, the word superiority should be observed with the first group but not the second. Directional attention shifts across the visual field have been induced by successive presentations of material in other studies (Chastain \& Ersoff, 1977; Shulman, Remington, \& McLean, 1979), even when the distance involved was as small as $.5 \mathrm{deg}$ of visual angle (Posner, 1978). In contrast, when different types of material are simultaneously presented, they may be processed in parallel (Keren, O'Hara, \& Skelton, 1977) and without interaction (even when one type bilaterally flanks another) when the two types are as different as the dots and letters used currently (Hepler, 1977; Wolford \& Morrison, Note 3).

\section{Method}

Subjects. Two groups of 10 subjects/group served for extra credit in a general psychology course. All reported normal or corrected-to-normal vision.
Apparatus. Stimuli were self-triggered by subjects for binocular viewing in a Scientific Prototype Model N-1000 three-channel manual tachistoscope. The luminance of each channel was approximately $130.0 \mathrm{~cd} / \mathrm{m}^{2}$, as measured by a Spectra Lumicon with Photospot attachment. A 40-W bulb shielded from the subject provided dim illumination for the laboratory.

Stimuli. Four trigrams, TAX, TUX, XAT, and XUT, each subtended a horizontal visual angle of $.64 \mathrm{deg}$. Letters were $.28 \mathrm{deg}$ high. The pre- and postexposure mask was composed of five superimposed $\mathrm{Xs}$ and $\mathrm{Os}$ with a horizontal line drawn contiguous to the top and bottom of the characters. The mask subtended a horizontal visual angle of $1.07 \mathrm{deg}$. Dots were small solid squares .11 deg on each side, which could lay .16 deg from the horizontal midline of an outside letter of the trigram. Trigrams were traced from a Berol Rapi Design lettering guide, $\mathrm{R}-2965$. All stimuli were drawn on white index cards in black ink with a Pilot Razor Point pen. The index cards were positioned on the Masonite slides so that the trigram would be centered behind the mask if both were shown simultaneously. Dots, when present, were centered behind the outside curve of the first or fifth $O$ in the mask.

Experimental design. Each trigram was presented for 24 criterion exposures to each subject. A dot appeared to the left, right, both left and right, and neither left nor right of the trigram six times each. Thirty-two practice exposures, with each possible combination of dots and trigrams repeated twice, preceded the criterion presentations. The order of appearance of the combinations was randomized for each subject, with the constraint that each combination appear once in each block of 16 exposures.

Procedure. Each subject was first familiarized with each trigram and dot combination. Subjects were told to report, first, the number of dots, and then, the identity of the middle letter of the trigram, after each exposure. For the first ("successive") group, the presentation began with either a dot to the left or a blank field for $50 \mathrm{msec}$. The preexposure mask then returned for $75 \mathrm{msec}$, followed by the appearance of the trigram, which possibly had a dot to its right. The postexposure mask then appeared. Subjects in this successive group were not explicitly told that the dot to the left would precede the trigram when present. For the other ("simultaneous") group, any $\operatorname{dot}(\mathrm{s})$ present always appeared simultaneously with the trigram. The duration for which each trigram was presented to each subject was initially $200 \mathrm{msec}$ and was reduced during the practice trials to allow about $75 \%$ overall accuracy in identifying the target letter by the beginning of the criterion presentations. Thereafter, the duration was adjusted only between blocks of 16 trials to maintain the subject's accuracy at about $75 \%$. The presentations proceeded in an uninterrupted series with an intertrial interval averaging approximately $10 \mathrm{sec}$. Subjects were given no feedback regarding accuracy and were not told that only the last 96 exposures were criterion presentations.

\section{Results and Discussion}

Mean proportion of identifications correct for each target in the word and nonword trigrams flanked by a dot to the left, right, both, and neither was calculated for each subject in each group. The resulting means were entered into an analysis of variance. The means for the significant interaction between groups and word/nonword context $[F(1,18)=7.38, p<.025]$ appear in Table 1 . No main effect and no other interaction was significant. ${ }^{1}$ Individual comparisons showed a WSE for the successive group $[\mathrm{t}(9)=2.95, \mathrm{p}<.025]$, but no WSE for the simultaneous group $[\mathrm{t}(9)=1.28]$. Data were reanalyzed after collapsing across the number of dots flanking the 
Table 1

Mean Proportion Correct With the Word and Nonword Context for the Groups Receiving the Left Dot and Trigrams Successively and Simultaneously in Experiment 1

\begin{tabular}{ccc} 
& \multicolumn{2}{c}{ Left Dot and Trigrams } \\
\cline { 3 - 3 } Context & Successively & Simultaneously \\
\hline Word $\left(\mathrm{T}_{-} \mathrm{X}\right)$ & .777 & .735 \\
Nonword $\left(\mathrm{X}_{-} \mathrm{T}\right)$ & .725 & .769 \\
\hline
\end{tabular}

strings with a correction for response bias (Gummerman, 1972). The significant interaction between groups and context persisted $[F(1,18)=6.42, p<.025]$, and individual comparisons again indicated the WSE only for the successive group $[t(9)=2.71, p<.025 ; t(9)=1.16$ for the simultaneous group].

An analysis of subjects' accuracy in counting dots showed no significant difference between the groups, with an overall mean accuracy of $77.7 \%$. For neither group was an error made on both the dots and target letter on the same exposure more frequently than would be expected by chance.

Mean trigram exposure durations did not differ for the two groups. The overall mean was $54.292 \mathrm{msec}$.

When subjects were required by the nature of the presentation to scan the letter arrays from left to right, a WSE emerged with a predesignated set of target letters appearing in a predesignated position in each array. However, another group who performed the same tasks showed no WSE when left-to-right scanning was not required. Lateral masking of the central target letter should have been the same for all arrays, since each letter was symmetrical across its vertical midline. Nevertheless, the sample of target letters and flankers was very small, and it seems desirable to test the generality of the finding with a much larger set of letter arrays. The following experiment was conducted to provide such a test.

\section{EXPERIMENT 2}

In the current experiment, each subject received a - block of presentations in which the target letter was the first letter of each trigram and another in which it was the last. Target letters were $\mathrm{M}, \mathrm{N}, \mathrm{P}$, and $\mathrm{R}$. In addition, one group of subjects was required to count the number of dots beside each trigram. The dot to the left, when present, always appeared before the trigram. The other group of subjects merely identified the target letter and received no dots. Subjects always knew which position the target letter would occupy in the letter array. Consonant with the results of Experiment 1, only the group of subjects receiving the dots was expected to show the WSE.

\section{Method}

Subjects. Two groups of eight subjects per group received extra credit in a general psychology course for their participation. All reported having normal or corrected-to-normal vision.
Apparatus. The apparatus and its luminance settings were identical to those in Experiment 1.

Stimuli. The 64 trigrams (shown in the appendix) were drawn in the same manner as those in Experiment 1. The size and positioning of the trigrams, dots, and masks were the same as in that experiment.

Experimental design. Each trigram was presented three times to each subject. The trigrams designated with an asterisk in the appendix were always shown with a dot to the right of the last letter to one group of subjects. To this group (the scanning group), a dot was shown to the left of each trigram on a randomly selected $50 \%$ of the presentations. The other group of subjects (the identification-only group) received the trigrams, but no $\operatorname{dot}(\mathrm{s})$. Half of each group received trigrams with the target letter in the first position for the first block of 96 criterion presentations. A separate set of practice trigrams was shown for 32 exposures before the first block, and another set with the target letter in the other position was shown for 32 exposures before the second block of 96 criterion presentations. The 16 word and 16 nonword trigrams for each criterion block were shown in a random order during the first 32 trials, although 8 of each were shown during the first 16 trials. The slides were shuffled before each of the two remaining series of 32 trials, and eight word trigrams were shown during each subseries of 16 trials.

Procedure. Each subject was first given a set of target letters and the critical position in the letter array for the first block. Subjects in the scanning group were instructed always to report the number of dots before the identity of the target letter, but they were not told that the left dot, when present, would precede the trigram. As in Experiment 1, for this group, the presentation began with either a dot to the left or a blank field for $50 \mathrm{msec}$, followed by a return of the preexposure mask for $75 \mathrm{msec}$, then the trigram and possibly a dot to its right, and then the postexposure mask. For the identification-only group, the trigram merely interrupted the mask. The practice trigrams were presented initially for $200 \mathrm{msec}$ to each subject, and the duration was reduced to allow about $75 \%$ accuracy in identifying the target letter by the beginning of the criterion trials in each block. The duration was thereafter adjusted only between subseries of 16 trials to maintain the subject's accuracy in identifying the target letter at about $75 \%$. The interruptions from changing slide sets, shuffling slides, and so on, were kept as brief as possible. Subjects were not told they were to be shown each slide more than once, and no feedback regarding accuracy was given.

\section{Results and Discussion}

Mean proportion of correct identifications for each target-letter position in word and nonword trigrams was calculated for each subject in each group. These means were entered into an analysis of variance. Performance was significantly better on word trigrams $[F(1,14)=$ $12.29 ; \mathrm{p}<.011$, and there was a significant interaction between groups and word/nonword context $[F(1,14)=$ $9.92, \mathrm{p}<.01]$. Individual comparisons showed that the significant interaction was due to better performance on words for the scanning group $[\mathrm{t}(7)=8.28, \mathrm{p}<.001]$, but not for the identification-only group $[t(7)=.19]$. Additional analyses showed a highly significant WSE when the target letter was first $[t(7)=5.87, p<.001]$ and when it was last $[t(7)=5.61, p<.001]$ for the scanning group, but for targets in neither position for the identification-only group $[t(7)=.20$ and $t(7)=.10$ for the first and last positions, respectively]. Means for the significant main effect and interaction appear in Table 2 . In addition, the total number of errors on each 
Table 2

Mean Proportion of Responses Correct With the Word and Nonword Contexts for Each Group in Experiment 2

\begin{tabular}{lccc}
\hline & \multicolumn{3}{c}{ Group } \\
\cline { 2 - 4 } Context & Scanning & $\begin{array}{c}\text { Identification } \\
\text { Only }\end{array}$ & Mean \\
\hline Word & .806 & .767 & .787 \\
Nonword & .733 & .763 & .748 \\
\hline
\end{tabular}

trigram for each group is indicated in the appendix. No other main effect or interaction was significant (all Fs $<1.0$ ).

The scanning group miscounted the dots on only $4.8 \%$ of the exposures. The error rate is lower than that for the corresponding group in Experiment 1, probably because the mean trigram exposure duration was significantly shorter in that experiment $[48.417 \mathrm{msec}$ vs. $88.359 \mathrm{msec}$ in the current experiment for the corresponding groups; $t(16)=2.72, p<.05$ ] . There was no significant difference in trigram exposure duration between the two groups in the current experiment.

The scanning group made an error on both the dot and target letter on the same exposure no more often than would be expected by chance. Analyses were conducted on the data from the scanning group to assess the effect on letter identification of the appearance of a dot with or before the trigram in conjunction with context and the position of the target letter. In the analysis of whether or not a dot appeared with the trigram, aside from the highly significant main effect of context, no main effect or interaction approached significance. When a dot appeared before the trigram, there was some tendency for accuracy to be lower than when one did not [proportions correct $=.742$ and .797 , respectively; $F(1,7)=4.83, .05<\mathrm{p}<.10$ ], although the effect did not interact with context $(F<1.0)$. Besides the main effect of context, no other main effect or interaction approached significance in the analysis of the effect of the dot preceding the trigram.

The results of the current experiment were quite similar to those of the first. With potential targets and their position predesignated, the WSE appeared only when scanning across the letter array was required.

\section{GENERAL DISCUSSION}

Both of the experiments just described give strong support to the hypothesis that holistic encoding of letter strings comprising words, which can be induced by scanning, plays an important role in the WSE. Subjects who were given tasks and presentations that made a leftto-right scan across the letter arrays advantageous showed the WSE. Those who were presented the same letter arrays in such a way that the inducement to scan was missing showed no such effect. The results were obtained with a fixed set of target letters appearing in the pre- designated positions in trigrams and were observed with targets in the first, middle, and last positions.

The present results are inconsistent with at least one class of possible explanations of the WSE. This type of explanation argues that uncertainty about the position of letters in the array is basic to the WSE. Visual confusion between the target and nontarget letters, coupled with incomplete extraction of the features of the letters in the string, is claimed to produce a situation in which a letter in a nontarget position is misidentified as an incorrect target. Orthographic constraints in words reduce the frequency of such positional errors, resulting in a WSE. This explanation was proposed by Estes (1975a, 1975b), and some researchers have interpreted their experimental results as supporting it. Appelman (1976) observed a WSE on same-different judgments of pairs of four-letter word and nonword strings. However, when the position of the crucial comparison was made unambiguous by removing all letters but one from one of the strings, the WSE disappeared. Paap and Newsome (1980b) eliminated the WSE by cuing the position of the target letter before each exposure. According to the positional uncertainty explanation, if the position of the target letter is known in advance, the WSE should disappear. The current results disconfirm that expectation and, instead, suggest that holistic encoding of word strings, which can result from scanning, is a crucial factor in the WSE. Lack of scanning by subjects can account for the results observed by Appelman (1976) and Paap and Newsome (1980b). Since the strings to be compared were presented one on top of the other in the Appelman (1976) study, with all but the critical letter removed from one of the strings there was no need to scan the other string to make the comparison. When Paap and Newsome (1980b) cued the target position before the exposure, this also eliminated the necessity of scanning. In the many studies revealing a WSE with the target position designated only after each exposure, subjects likely scanned each letter array in a left-to-right manner, as they might when reading.

\section{REFERENCE NOTES}

1. McClelland, J. L., \& Rumelhart, D. E. An interactive activation model of the effect of context in perception, Part I (Report No. 8002). La Jolla: University of California, San Diego, Center for Human Information Processing, 1980.

2. Rumelhart, D. E., \& McClelland, J. L. An interactive activation model of the effect of context in perception, Part II (Report No. 8003). La Jolla: University of California, San Diego, Center for Human Information Processing, 1980.

3. Wolford, G., \& Morrison, F. J. Levels of processing of unattended input. Paper presented at the annual meeting of the Psychonomic Society, Denver, Colorado, November 1975.

\section{REFERENCES}

Appelman, I. B. The word superiority effect: Dependence on short-term memory factors. Memory \& Cognition, 1976, 4, 156-161. 
Baron, J., \& Thurston, I. An analysis of the word-superiority effect. Cognitive Psychology, 1973, 4, 207-228.

Carr, T. C., Lehmkuhle, S. W., Kottas, B., Astor-Stetson, E. C., \& ARNolD, D. Target position and practice in the identification of letters in varying context: A word superiority effect. Perception \& Psychophysics, 1976, 19, 412-416.

Chastain, G. Feature analysis and the growth of a percept. Journal of Experimental Psychology: Human Perception and Performance, 1977, 3, 291-298.

Chastain, G. Phonological and orthographic factors in the wordsuperiority effect. Memory \& Cognition, 1981, 9, 389-397.

Chastain, G., \& Ersoff, J. Effect of cumulative directional attention shifts on the comparison and integration of visual stimuli. Journal of Experimental Psychology: Human Perception and Performance, 1977, 4, 641-652.

Estes, W. K. The locus of inferential and perceptual processes in letter identification. Journal of Experimental Psychology: General, 1975, 104, 122-145. (a)

Estes, W. K. Memory, perception and decision in letter identification. In R. L. Solso (Ed.), Information processing and cognition: The Loyola symposium. Potomac, Md: Erlbaum, 1975. (b)

Greengerg, S. N., \& KrUeger, L. E. Limitations on the wordsuperiority effect with a fixed target set. Bulletin of the Psychonomic Society, 1980, 15, 25-28.

Guilford, J. P., \& Fruchter, B. Fundamental statistics in psychology and education (6th ed.). New York: McGraw-Hill, 1977.

Gummerman, K. A response-contingent measure of proportion correct. Journal of the Acoustical Society of America, 1972, 52, 1645-1647.

Haber, R. N., \& Hershenson, M. Effects of repeated brief exposures on the growth of a percept. Journal of Experimental Psychology, 1965, 69, 40-46.

HePLe R, S. P. Selective information processing. Memory \& Cognition, $1977,5,449-452$.

JACEWICZ, M. M. Word context effects on letter recognition. Perceptual and Motor Skills, 1979, 48, 935-942.

Johnson, N. F., \& MARMUREK, H. H. C. Identification of words and letters within words. American Journal of Psychology, $1978,91,401-415$.

Johnston, J.C., \& McClelland, J. L. Perception of letters in words: Seek not and ye shall find. Science, 1974, 184, 11921194.

Keren, G., O'Hara, W. P., \& Skelton, J. M. Levels of noise processing and attentional control. Journal of Experimental Psychology: Human Perception and Performance, 1977, 3, 653664.

LAWRY, J. A., \& LABERGE, D. Letter and word code interactions elicited by normally displayed words. Perception \& Psychophysics, 1981, 30, 71-82.

Manelis, L. The effect of meaningfulness in tachistoscopic word perception. Perception \& Psychophysics, 1974, 16, 182-192.

Massaro, D. W. Perception of letters, words, and nonwords. Journal of Experimental Psychology, 1973, 100, 349-353.

McClelland, J. L. On the time relations of mental processes: An examination of systems of processes in cascade. Psychological Review, 1979, 86, 287-330.

Mewhort, D. J. K., \& BeAL, A. L. Mechanisms of word identification. Journal of Experimental Psychology: Human Perception and Performance, 1977, 3, 629-640.

Mewhort, D. J. K., Merikle, P. M., \& Bryden, M. P. On the transfer from iconic to short-term memory. Journal of Experimental Psychology, 1969, 81, 89-94.

PaAP, K. W., \& Newsome, S. L. Do small visual angles produce a word-superiority effect or differential lateral masking? Memory \& Cognition, 1980, 8, 1-14. (a)

PaAP, K. W., \& Newsome, S. L. A perceptual-confusion account of the WSE in the target search paradigm. Perception \& Psychophysics, 1980, 27, 444-456. (b)
Posner, M. I. Chronometric explorations of mind. Hillsdale, N.J: Erlbaum, 1978.

Purcell, D. G., Stanovich, K. E., \& Spector, A. Visual angle and the word-superiority effect. Memory \& Cognition, 1978, 6, 3-8.

Shulman, G. L., Remington, R. W., \& Mclean, J. P. Moving attention through space. Journal of Experimental Psychology: Human Perception and Performance, 1979, 5, 522-526.

Smith, E. E., \& Spoenr, K. T. The perception of printed English: A theoretical perspective. In B. H. Kantowitz (Ed.), Human information processing: Tutorials in performance and cognition. Potomac, Md: Erlbaum, 1974.

Spector, A., \& Purceli, D. G. The word-superiority effect: A comparison between restricted and unrestricted alternative sets. Perception \& Psychophysics, 1977, 21, 323-328.

\section{NOTE}

1. Following the guidelines described by Guilford and Fruchter (1977), a difference described as not significant indicates $\mathrm{p}>.10$.

Appendix

Word (Orthographic) and Nonword Trigrams With Total Number of Errors on Each for the Scanning (S) and Identification-Only (I) Groups in Experiment 2

\begin{tabular}{|c|c|c|c|c|c|}
\hline Word & $\mathrm{S}$ & I & Nonword & $S$ & I \\
\hline MAG & 4 & 8 & MGA* & 9 & 6 \\
\hline MED* & 3 & 1 & MDE & 5 & 4 \\
\hline MOB & 5 & 7 & MBO* & 5 & 5 \\
\hline MUT* & 6 & 9 & MTU & 4 & 5 \\
\hline NAG* & 3 & 9 & NGA & 11 & 12 \\
\hline NED & 4 & 6 & $\mathrm{NDE}^{*}$ & 10 & 6 \\
\hline NOB* & 7 & 5 & NBO & 1 & 8 \\
\hline NUT & 6 & 9 & $\mathrm{NTU}^{*}$ & 7 & 6 \\
\hline PAG & 2 & 2 & PGA* & 6 & 2 \\
\hline PED* & 5 & 5 & PDE & 6 & 1 \\
\hline POB* & 3 & 5 & PBO & 8 & 3 \\
\hline PUT & 3 & 5 & PTU* & 3 & 5 \\
\hline RAG* & 2 & 5 & RGA & 5 & 7 \\
\hline RED & 3 & 4 & $\mathrm{RDE}^{*}$ & 5 & 5 \\
\hline ROB & 6 & 6 & $\mathrm{RBO}^{*}$ & 7 & 7 \\
\hline RUT* & 7 & 2 & RTU & 9 & 8 \\
\hline $\mathrm{JAM}^{*}$ & 6 & 7 & $\mathrm{AJM}$ & 5 & 4 \\
\hline HEM & 3 & 6 & EHM $^{*}$ & 10 & 3 \\
\hline SOM* & 12 & 6 & OSM & 7 & 4 \\
\hline BUM & 7 & 2 & UBM $^{*}$ & 9 & 6 \\
\hline JAN & 4 & 8 & $\mathrm{AJN}^{*}$ & 9 & 7 \\
\hline HEN* & 5 & 4 & EHN & 5 & 8 \\
\hline SON & 5 & 4 & OSN* & 7 & 7 \\
\hline BUN* & 2 & 5 & UBN & 6 & 8 \\
\hline JAP* & 5 & 4 & AJP & 6 & 5 \\
\hline HEP & 2 & 3 & EHP* & 5 & 4 \\
\hline SOP & 3 & 3 & OSP* & 2 & 1 \\
\hline BUP* & 4 & 5 & UBP & 6 & 3 \\
\hline JAR & 6 & 10 & $\mathrm{AJ} \mathrm{R}^{*}$ & 7 & 7 \\
\hline HER* & 8 & 9 & EHR & 7 & 7 \\
\hline SOR* & 2 & 9 & OSR & 5 & 8 \\
\hline BUR & 6 & 6 & UBR* & 8 & 10 \\
\hline
\end{tabular}

*Trigram always had a dot to its right when it was shown to Group $S$.

(Received for publication June 4, 1981; revision accepted February 2, 1982.) 